\title{
Peer Influence in Network Markets: An Empirical and Theoretical Analysis
}

\author{
Joachim Henkel ${ }^{1,2}$, Jörn Block ${ }^{1}$ \\ January 2008 \\ (earlier version: January 2007) \\ Draft, comments welcome
}

\begin{abstract}
Network externalities spur the growth of networks and the adoption of network goods in two ways. First, they make it attractive to join a network with a large installed base. Second, they make it attractive for network members to actively recruit new members. Despite indications that this "peer effect" can be more important for network growth than the installed-base effect, it has so far been largely ignored in the literature. We aim at closing this gap, using both survey data and a game-theoretical model. Comparing respondents' adoption of two distinct networks goods, we find that the peer effect matters strongly for small, but not for large networks. Results from model analysis support this finding. Under conservative assumptions, the increase in network size due to the peer effect is by an additive constant - which, for small networks, can amount to a large relative increase. The difference between small, local, and personal networks and large, global, anonymous networks thus arises endogenously from our model. In the duopoly case, we find that introducing the peer effect favors winner-take-all outcomes. We use the examples of the Internet services, Skype and eBay, for our empirical analysis and as illustration of our theoretical findings. Since many network goods give rise to small, local networks, our findings are highly relevant for the management of network goods and the social networks they can give rise to.
\end{abstract}

Keywords: Network Markets; Peer Influence; Diffusion; Technology Adoption

\footnotetext{
${ }^{1}$ Schöller Chair in Technology and Innovation Management, Technische Universität München, Arcisstr. 21, D - 80333 Munich, Germany. Phone: +49 - 89 - 289 25741, fax: +49 - 89 - 289 25742, email: block@wi.tum.de, henkel@wi.tum.de.

${ }^{2}$ Centre for Economic Policy Research (CEPR), London.
} 


\section{Introduction}

For many products a user's utility depends on the number of other users of the good (Katz and Shapiro 1985). Such network externalities have become ubiquitous with the information economy, in particular with the rise of Instant Messaging services and virtual communities (Liebowitz 2002, p. 20-22). For the diffusion of new goods, network externalities have two rather different implications. First, the more users a good has, the more attractive the good becomes for potential further adopters. We call this the installed-base effect since it is caused by the attractiveness of the current user base to outsiders. Second, growth of the installed base is beneficial also to those consumers who are already part of it. They thus have an incentive to support its growth by exerting influence on not-yet adopters. We refer to this second mechanism as the peer effect. Since current users will exert influence mainly on those outsiders to which they maintain social ties, the peer effect relates social networks to the diffusion of network goods (with a slightly different meaning of the term "network"). In the following, we refer to the installed base of the network good as the network under consideration, in contrast to a social network. While the installed-base effect has been treated extensively in the literature (e.g., Katz and Shapiro 1986, 1992), the peer effect has received very little attention. ${ }^{3}$ The purpose of this paper is to fill this gap by providing empirical evidence, a theoretical analysis, and management implications of the peer effect.

As an illustration consider the case of Skype, a proprietary peer-to-peer Internet telephony (VoiP) network. ${ }^{4}$ The first public version of Skype was released in August 2003. In October 2004, the peak number of subscribers simultaneously logged on to Skype reached 1 million; by January 2008, this number had grown beyond 11 million. The total number of registered users had exceeded 100 million already by April 2006. ${ }^{5}$ On its website, the provider of Skype gives a good example of how individuals drag their peers into adopting the service, and thereby support growth of the network as a whole. Under the category Skype stories, a user describes how he tries to organize a rehearsal of his reunioned college band: "I'd been using Skype for a while by that time. I got everyone else signed up for

\footnotetext{
${ }^{3}$ The studies by Domingos and Richardson (2001) and Subramani and Rajagopalan (2003) are two exceptions published in the field of computing and information systems. The latter article in particular addresses, in a conceptual model, the motives of network members to recruit further adopters.

${ }^{4}$ With some simplification, Skype works in the following way. After registering with the Skype service, a user builds her personal contact list by sending contact requests to other users, or receiving and accepting requests herself. She can then call each person on her personal contact list via Skype. Calls are transmitted over the internet, and are free of charge for both parties. More recent versions of the Skype software also include other features such as conference calls, calls to fixed-line phones (SkypeOut), sending SMS or Instant Messaging. In September 2005, Skype was acquired by the Internet auction provider eBay.
}

${ }^{5}$ See http://share.skype.com/sites/en/2008/01/splinters_all_around_breaking.html and http://share. skype.com/sites/en/2006/04/skype_hits_100_million_users_c.html (accessed 01/12/2008). 
it, even our bass player who isn't the most technically adept." ${ }^{6}$ An early adopter thus persuaded his friends to join the network, possibly also providing them with necessary information and support. The network as a whole grew by the number of his fellow band members who might in turn persuade their friends to also join the network. In principle, such activity is well known as viral or word-of-mouth marketing. The distinctive feature of the band reunion example, however, is that the focal person was motivated by his own benefit deriving from network externalities. The quality and level of this motivation distinguishes the (network externality-based) peer effect clearly from other drivers of viral marketing. The marketing department at Skype is aware of the power of this effect, and supports this particular peer-based way of diffusion. On its website, the provider of Skype offers users the possibility to inform their friends about Skype. The pre-formulated wording of the e-mail is "Skype is free to download and easy to install. Once you have it you can call me and other Skype users for free." 7

The example above highlights yet another point central to our study. The value of Skype for the focal user strongly depends on how many of his fellow musicians have adopted the service. In contrast, most of the other millions of Skype users are irrelevant to him. That is, the Skype network that matters for him is a replication of an existing - small and local - social network, not the universe of all Skype users. Accordingly, the strength of the network effects at work is determined by the size of his personal network, not by the total number of adopters of the service.

The type of "local" network externality described above is in stark contrast to that present, e.g., in marketplaces. Consider the case of the online auction service eBay. For someone intending to sell a good to a friend there would be little need to conduct the transaction over eBay. The value of eBay, flee markets, and similar marketplaces lies precisely in the fact that they match strangers with corresponding desires to sell and to buy. Unlike Skype, they are not based on existing ties, but aim at creating new ones. They are global, non-personal, and typically large. Thus, apart from standard word-ofmouth marketing it makes little sense for an eBay user to talk her friends into adopting the service.

We set out to analyze the peer effect described above using both a survey and a gametheoretical model, the latter employing the concept of fulfilled expectation equilibria. Building upon Hotelling's (1929) classical approach we first analyze the monopoly case. We present three ways of modelling peer influence, arguing that the most conservative assumptions are also the most realistic ones. Under these assumptions, adopters of the good coordinate in small groups of size $n$ (where $n=1$ is particularly important) to sponsor further adopters. Such sponsoring may take the form of advice or technical

\footnotetext{
${ }^{6}$ See http://www.skype.com/share/stories/a-band-reunion-through-skype (accessed 01/12/2008).

${ }^{7}$ See http://www.skype.com/share/tellafriend (accessed 01/12/2008).
} 
support, in which case one unit of cost (or effort) expanded by the sponsors translates into several $(\kappa)$ units of benefit for the sponsored person. We refer to $\kappa$ as the cost leveraging factor.

Results for the monopoly case are that introducing peer influence leads to an increase in equilibrium network size which depends positively on the group size $n$, the cost leveraging factor $\kappa$, and the relative strength of the network externality. When the provider charges for the network good, equilibrium prices are higher than in the base case absent peer influence; that is, the seller partly skims off the increased attractiveness of her good. Most importantly, we find that the increase in network size is by an additive constant, as opposed to a factor. This implies that a sizeable relative growth, compared to the base case, can be attained for small, but not for large networks.

The difference between the two types of networks sketched above thus arises endogenously from our model: the effective personal network of most Skype users is small, and growing one's personal network by exerting peer influence makes sense. In contrast, all eBay users - in sufficient geographical proximity - are potential buyers of the good a person offers. Growing this already huge network by exerting peer influence makes little sense.

In the duopoly case, two additional questions arise. First, how does the peer effect influence winner-take-all outcomes? Does it favor asymmetric equilibria, or does it render a symmetric situation more stable? Second, is price competition intensified or mitigated by the peer effect? We model duopolistic competition using a two-dimensional extension of Hotelling's model, in order to make total demand price elastic. Regarding stability of symmetric solutions we find that, under most circumstances, introducing the peer effect favors winner-take-all outcomes. As to pricing, two counteracting effects are present: a bias towards higher prices, as described above for the monopoly case, and tougher competition due to increased overall market size. It depends on the parameter values which effect prevails.

Finally, we present data from a large-scale survey on the adoption of Skype and eBay. Very clearly, our data support the model results. For Skype, what drives individuals to adopt the service are the peer effect and the local installed base effect (i.e., the effect of the installed base among the members of the potential adopter's pre-existing social network). For eBay, in contrast, both of these effects are of little relevance, while the global installed base effect drives adoption.

The remainder of the paper is organized as follows. We discuss the related literature in Section 2. Sections 3 and 4 present the analysis of the monopoly case, from the adopter's and the seller's perspective, respectively. Duopoly is treated in Section 5. Finally, we present our empirical data in Section 6 and, in Section 7, discuss our findings and point out open questions. 


\section{Related Literature}

The analysis of the diffusion of network products dates back to work by Katz and Shapiro (1985, 1986) and Farrel and Saloner $(1985,1986)$. A large strand of literature has built on these early contributions. Relevant in our context are publications on local network externalities as well as a number of empirical studies.

Some theoretical articles from the field of evolutionary economics focus on local rather than global network externalities. Positive externalities from adoption of a network good are no longer assumed to encompass the entire population, but only a small subset of it. "Local" in this context denotes social rather than geographical proximity: local network externalities are present between individuals who interact more frequently or more intensively with each other than with persons outside the "local" network (Koski and Kretschmer 2004). Cowan and Miller (1998) as well as Jonard and Yildizoglu (1998) show that these local externalities are a reason for non-standardization: winner-take-all outcomes do prevail within each local network, but different goods may win in different local networks. On a global scale, thus, standardization may not arise. The existence of languages and local dialects is an illustrative example. There still exist languages or local dialects that are only spoken by a few people, in some extreme cases less than ten. ${ }^{8}$ As Church and King (1993) show, this outcome can not prevail under the assumption of global network externalities. Abrahamson and Rosenkopf (1997) analyze the effect of social network structure on the extent of innovation diffusion, assuming that information about the innovation is channeled by social networks. In a similar vein, Deroïan (2002) develops a model of innovation diffusion with interacting agents, who influence each other in their effort to evaluate the innovation.

Our approach differs from earlier work on local, or social network-based, network effects by explicitly modelling peer influence - that is, the possibility that adopters can influence, or even subsidize, those having not yet adopted in order to increase their own utility derived from network externalities. This mechanism has largely been ignored in the literature, the studies by Subramani and Rajagopalan (2003) and Domingos and Richardson (2001) being two exceptions. Subramani and Rajagopalan (2003) described the peer effect as "motivated evangelism" in a conceptual, qualitative model. Domingos and Richardson (2001) analyze, theoretically and empirically, the "network value" of a customer, which arises from her influencing others to buy. With "network value", however, refering to the underlying social network rather than to network externalities, they do not focus explicitly on the influencer's motivation based on to her internalizing network externalities. By addressing this source of motivation explicitly in both model and empirical analysis, our study goes beyond these papers. Furthermore, the distinction

\footnotetext{
${ }^{8}$ For a more detailed description and some references on these languages see Koski and Kretschmer (2004).
} 
between small (and thus local) and large (and thus global) networks is not built into our assumptions, but arises endogenously in the interpretation of our findings.

On the empirical side, there is some extant work on the installed-base effect, but very little on the peer effect. The installed-base effect is found to exist in industries as diverse as PC and software (e.g., Brynjolfsson and Kemerer 1996; Gandal 1994; Koski 1999), fax machines (Economides and Himmelberg 1995) automated teller machines (Saloner and Shepard 1995), telecommunications (Majumdar and Venkatamaran 1998), consumer electronics (Shankar and Bayus 2003), and yellow pages (Rysman 2004). To our knowledge, only one recent empirical study analyzed the peer effect (Block and Köllinger 2006). In this study, the authors show the peer effect to have a strong impact on the adoption of Internet-based Instant Messaging services.

Finally, it should be noted that the peer effect must be distinguished from wordof-mouth communication. ${ }^{9}$ Word-of-mouth describes a communication channel, without specifying the motive of the person exerting interpersonal influence. For example, a person might tell a friend about a recently acquired gadget out of pure enthusiasm. She might derive some utility from speaking to an intrigued listener, and possibly from the fact that her addressee copies her behavior by purchasing the same good. Her utility gain would then be linked either to the act of persuading itself or to a confirmation of her own behavior. It thus can be present for all types of goods, irrespective of network externalities. In contrast, the peer effect, as it is defined in this paper, implies that by recruiting a new user a network member realizes a continuous flow of utility which is directly linked to her use of the good. Peer influence may happen by means of wordof-mouth communication, but other means (e.g., technical support) are conceivable as well. Conversely, word-of-mouth communication may have the purpose of exerting peer influence, but this need not be the case.

\section{Monopoly: Adopters' Perspective}

\subsection{Adoption Without Peer Influence}

Following Hotelling's classical model (1929), we consider a market of heterogeneous consumers who are distributed on the positive real axis $[0 ; \infty)$ with density one. There is one good, offered by a monopolist at price $p$. If consumer $x$ was the only adopter of the good, she would derive a stand-alone utility of $u(x)$ from it, where $u(x)$ is twice continuously differentiable and $u^{\prime}(x) \leq 0$. In addition, her utility depends on the number

\footnotetext{
${ }^{9}$ Word-of-mouth communication is a much researched topic in marketing and communication research. For an early work in the field of communication research see Lazarsfeld et al. (1944), who introduce the distinction between word-of-mouth communication and mass media influence in the context of voter behavior. For an early contribution in the field of marketing see Arndt (1967), who analyzes the effect of word-of-mouth communication on the diffusion of a new product in an experimental setting.
} 
of other adopters. With a network size of $y$ her utility, and hence willingness to pay, is $u(x)+v(y)$. The network externality function $v(y)$ is twice continuously differentiable, increasing in $y$, and weakly concave: $v^{\prime}(y)>0, v^{\prime \prime}(y) \leq 0$. Furthermore, we make the obvious assumption that $v(1)=0$. When all consumers expect the future network size to be $y^{e x p}$ and base their adoption decision on this expectation, then the resulting network size $y$ (equal to the position of the marginal adopter) is given by $u(y)+v\left(y^{e x p}\right)-p=0$. A fulfilled expectations equilibrium (FEE) $y_{0}^{*}$ then is a solution to the following equation:

$$
u\left(y_{0}^{*}\right)+v\left(y_{0}^{*}\right)-p=0
$$

The consumers in $\left[0 ; y_{0}^{*}\right]$ adopt because they are attracted by the (expected) size of the installed base. That is, their adoption is driven by the installed base effect. We will refer to this outcome as the base case. Note that, depending on the shape of the functions $u$ and $v$ there may be multiple FEEs.

In order to exclude the (unrealistic) limiting case of an infinite number of adopters we require that $\lim _{y \rightarrow \infty}(u(y)+v(y))<0 .{ }^{10}$ This implies that, for each non-negative price $p$, the set of solutions to (1) has an upper bound. Existence of at least one FEE follows from the fact that $u(y)+v(y)-p$ is continuous and negative for sufficiently large $y$. So, either it is negative for all $y \geq 0$, in which case $y_{0}^{*}=0$ is the only equilibrium, or it is positive for some $y .{ }^{11}$ In the latter case, $u(y)+v(y)-p$ must change its sign from positive to negative somewhere, which implies existence of a stable equilibrium. ${ }^{12}$ In the following, we will use a linear functional form for $u$ and $v$ in order to simplify the analysis and to arrive at more concrete results:

$$
u(x)=u_{0}-\lambda x, \quad v(y)=\alpha y \quad(\text { linear case })
$$

In the linear case, the solution to equation (1) becomes

$$
y_{0}^{*}=\frac{u_{0}-p}{\lambda-\alpha} .
$$

The denominator reflects the relative strength of the network effect $(\alpha)$ compared to the

\footnotetext{
${ }^{10} \mathrm{~A}$ negative stand-alone utility of adoption may seem at odds with the usual assumption of "free disposal." However, adopting the good requires more than just purchasing it, since the consumer needs to install it and learn how to use it. These activities require effort and thus create cost.

${ }^{11} \mathrm{~A}$ third possibility would be that the function equals zero at one or more points and is negative otherwise. These roots would constitute equilibria which are unstable against deviations to lower values of $y$. We refrain from pursuing this case further since we focus on stable equilibria.

${ }^{12}$ A solution $y$ to equation (1) at which the sign changes from negative to positive would mean that, after a small deviation to $y-\epsilon<y$, the marginal consumer would experience a negative net utility and would hence not join the network, reducing its size further. In contrast, a positive deviation of the network size to $y+\epsilon$ would imply that the marginal non-consumer would derive a positive utility from joining the network. She would consequently do so and increase the network size further.
} 
utility loss from not having one's optimal product $(\lambda)$. When the network externality dominates $(\alpha \rightarrow \lambda)$, yo diverges and every consumer adopts. While it might indeed happen that a network good is adopted throughout the market, this limiting case is less interesting for our analysis. We thus assume that $\alpha<\lambda$.

\subsection{Maximum Adoption by Surplus Re-Distribution}

Given that all consumers in $\left[0 ; y_{0}^{*}\right]$ adopt the good, any additional adopter at $x>y_{0}^{*}$ would experience a negative net benefit. However, her adoption would increase the network size, benefitting all other adopters. If the network externality is strong enough and her disutility not too large, the overall effect of her adoption on consumer welfare will be positive. That is, with a suitable re-distribution of surplus between adopters a Pareto improvement compared to the outcome described in Section 3.1 can be achieved. We refer to the individuals on the giving end as "sponsors." When the adopter at $x$ adopts due to such sponsoring, then the adoption was based on the peer effect. Of course, the adopter takes also the size of the installed base into account. However, it is the peer effect which triggers her adoption decision.

In real life, peer influence may take the form of side payments. This is particularly plausible when the constituents of the network are firms. In this case, one unit of utility provided by a sponsor corresponds to one unit of utility received by the new adopter. However, peer influence may also be exerted in a non-monetary way. In fact, in networks of individuals this will be the norm rather than the exception. For example, a user of a certain software package may assist her friend in installing the software and learning how to use it. In that case, a high benefit for the new adopter can be generated with relatively little effort on the sponsor's side. For that reason, we introduce a "cost leveraging" parameter $\kappa$ : one unit of effort by a sponsor translates into $\kappa$ units of benefit for the sponsored individual. Our argument implies that $\kappa$ will in general be larger than unity.

Following the above consideration, we ask the following question. What is the maximum $y_{1}^{*}$ such that, if all consumers in $\left[0 ; y_{1}^{*}\right]$ adopt, total consumer surplus is not smaller than when only those in $\left[0 ; y_{0}^{*}\right]$ adopt? The question implies that, after a suitable redistribution of surplus (i.e., after peer effects came into play), no individual is worse off than in the base case.

When $y_{1}>y_{0}^{*}$ consumers adopt, then the net benefit of adoption (before re-distribution) is positive for all $x<\hat{y}_{1}$, where

$$
\hat{y}_{1}=u^{-1}\left(p-v\left(y_{1}\right)\right)
$$

Obviously, when $y_{1}>y_{0}^{*}$ and the FEE is unique, then $y_{0}^{*}<\hat{y}_{1}<y_{1}$. The sought-for value 


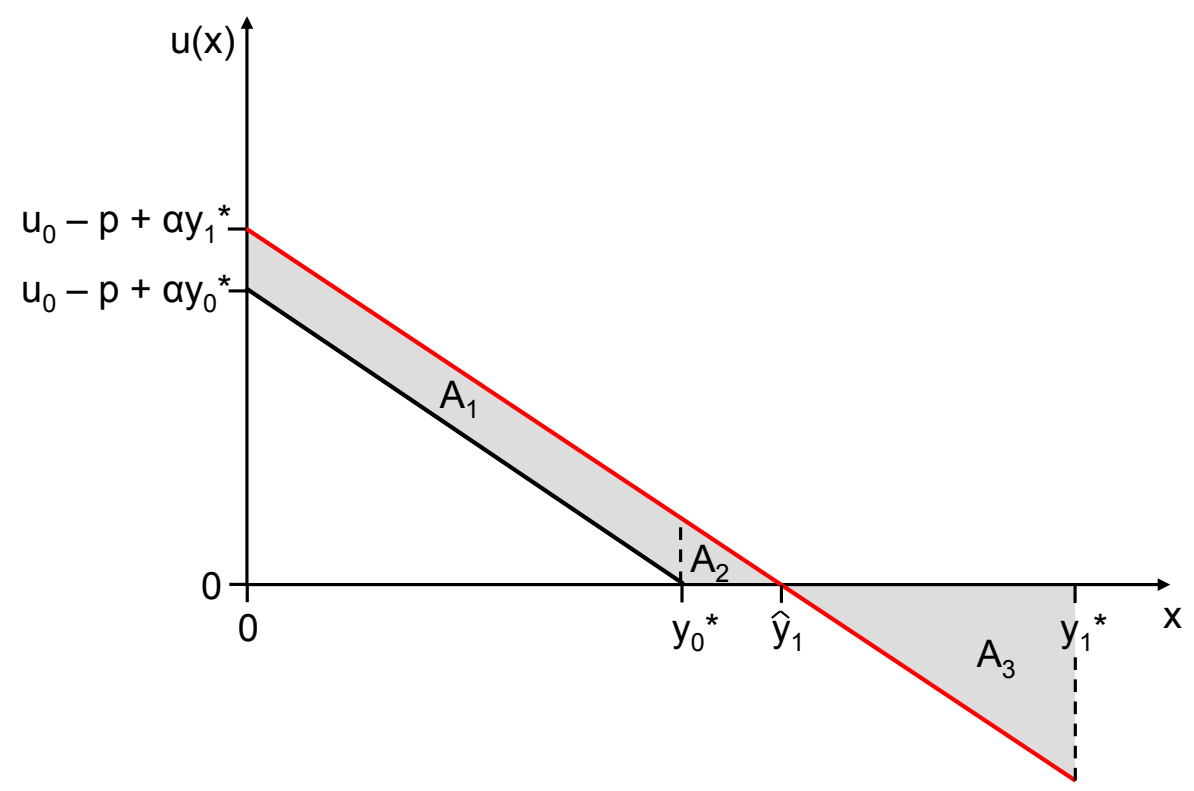

Figure 1: Network growth by peer effect and surplus re-distribution.

$y_{1}^{*}$ is then given by the following equation.

$$
y_{0}^{*}\left(v\left(y_{1}^{*}\right)-v\left(y_{0}^{*}\right)\right)+\int_{y_{0}^{*}}^{\hat{y}_{1}}\left(u(x)+v\left(y_{1}^{*}\right)-p\right) d x+\frac{1}{\kappa} \int_{\hat{y}_{1}}^{y_{1}^{*}}\left(u(x)+v\left(y_{1}^{*}\right)-p\right) d x=0
$$

The first two terms in this expression are positive. The first one describes the utility gains, due to the growth of the network from $y_{0}^{*}$ to $y_{1}^{*}$, of those consumers who also adopt in the base case. Figure 1 illustrates the argument for the case of $\kappa=1$, with the area $A_{1}$ corresponding to the first term in (5). The second term, corresponding to area $A_{2}$, captures the positive utility of those "new" adopters who are positioned below $\hat{y}_{1}$ and who thus have a positive net benefit of adoption without receiving any transfers. The last one, finally, is negative, and corresponds to area $A_{3}$. In order to capture the leveraging effect discussed above it carries the inverse of $\kappa$ as a factor.

In the linear case, $\hat{y}_{1}$ obtains as $\hat{y}_{1}=\left(u_{0}-p+\alpha y_{1}^{*}\right) / \lambda$. We introduce $\omega \equiv \alpha / \lambda$, measuring the relative strength of the network externality compared to the decrease in consumers' stand-alone utility when $x$ increases. Straightforward calculation yields the solution $y_{1}^{*}$ to equation (5):

$$
y_{1}^{*}=y_{0}^{*}\left(1+\frac{2 \omega \kappa}{1-2 \omega-\omega^{2}(\kappa-1)}\right)
$$

As in the base case, the network size diverges when the relative strength of the network effect exceeds a certain limit: for $\omega \rightarrow(\sqrt{\kappa}+1)^{-1}, y_{1}^{*}$ goes to infinity. In order to illustrate equation (6), Figure 2 depicts $y_{1}^{*} / y_{0}^{*}$ as a function of $\omega$, for various values of the leveraging parameter $\kappa$. The figure shows that even for modest values of the relative strength $\omega$ of the network externality and the cost leveraging factor $\kappa$, a considerable growth of the 


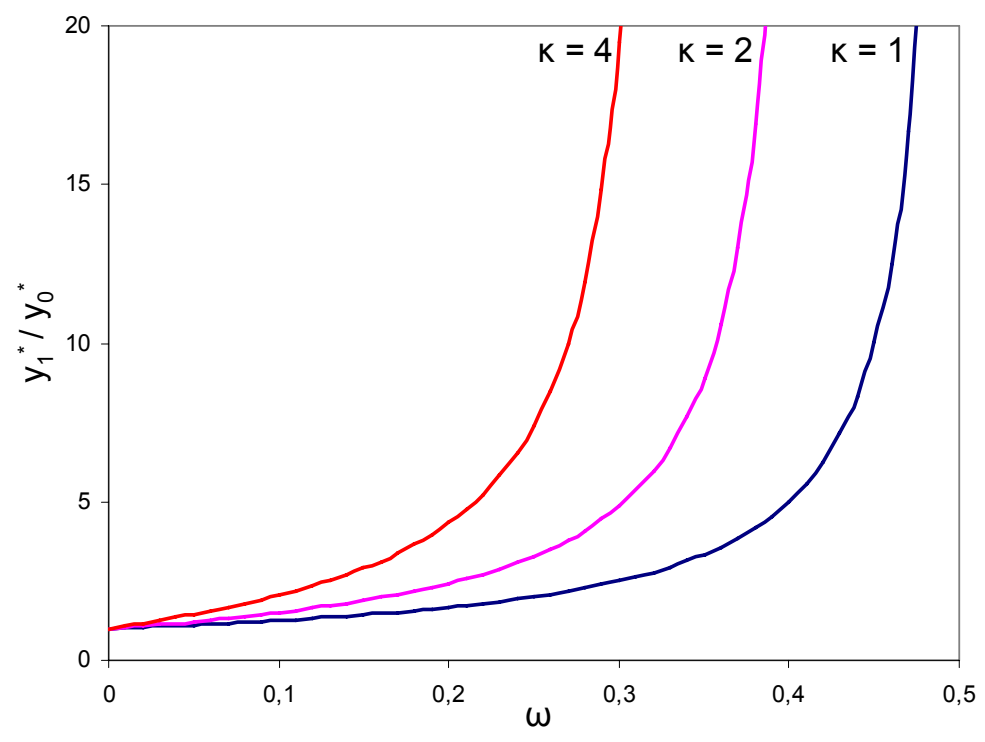

Figure 2: Relative network growth $y_{1}^{*} / y_{0}^{*}$ due to peer effect, see (6).

network is achieved. For example, with $\omega=0.28$ and $\kappa=2$, the network size $y_{1}^{*}$ is 4.1 times as large as in the base case.

For the general (i.e., non-linear) case, the following results can be shown. If $y_{0}^{*}>0$ exists then either a finite solution $\left(\hat{y}_{1}, y_{1}^{*}\right)$ to equations (4) (with $\left.y=y_{1}^{*}\right)$ and (5) exists, or the left-hand side of equation (5) is positive for all pairs $\left(\hat{y}_{1}, y_{1}^{*}\right)$, implying adoption by the whole market. This is true due to the facts that the l.h.s. of (5) is continuous in $y_{1}^{*}$ and that it is positive for values of $y_{1}^{*}$ incrementally larger than $y_{0}^{*}$. As to the number of equilibria, even if $y_{0}^{*}$ is unique there may be multiple solutions to equation (5).

\subsection{Benefit-Maximizing Coordinated Peer Influence}

So far, we have analyzed a (Pareto-neutral) re-distribution that leads to maximum adoption. This simplified scenario illustrates the power of the peer effect, and serves as a benchmark. We now move closer to a realistic situation by allowing the sponsors to maximize their own utility. There are two stages. In stage one, consumers decide about adopting the good without taking possible peer effects into account. ${ }^{13}$ They thus arrive at the base case described in Section 3.1. In stage two, the "early adopters" (i.e., those having adopted in stage one) coordinate to sponsor new adopters. They do so in such a way as to maximize their own utility.

Let the marginal consumer after the second stage be denoted by $y_{2}$. Then, as in equation $(5)$, we have a range of consumers $\left[y_{0}^{*}, \hat{y}_{2}\right]$ who do not require any sponsoring

\footnotetext{
${ }^{13}$ That is, they have realistic expectations concerning the adoption that will take place in stage one, but are myopic with regard to peer effects coming into play in stage two. Without this assumption, some consumers who do adopt in the base case would refrain from doing so, because they do better by deferring their adoption until the second stage, thus not being a sponsor. We will relax the assumption later on that each early adopter becomes a sponsor in stage two.
} 
because their net benefit of adoption is positive just due to the increase in network size from $y_{0}^{*}$ to $y_{2}$. In contrast to the analysis in Section 3.2 (maximum adoption) we assume here that they keep their surplus (instead of having it re-distributed to some other, more "needy" consumer). ${ }^{14}$ Thus, utility re-distribution takes place only between sponsors and those new adopters who are situated in $\left[\hat{y}_{2}, y_{2}\right]$. Assuming that the sponsors increase each new adopter's utility to the threshold level of zero, their aggregate utility increase $\Delta U_{E A}$ is given by the following equation.

$$
\Delta U_{E A}=y_{0}^{*}\left(v\left(y_{2}\right)-v\left(y_{0}^{*}\right)\right)+\frac{1}{\kappa} \int_{\hat{y}_{2}}^{y_{2}}\left(u(x)+v\left(y_{2}\right)-p\right) d x
$$

In this equation, $\hat{y}_{2}$ as a function of $y_{2}$ is given by an equation analogous to (4). As in (5), $\kappa$ takes account of the leveraging effect of the sponsors' effort. In the linear case, $\Delta U_{E A}$ obtains as

$$
\Delta U_{E A}=\alpha y_{0}^{*}\left(y_{2}-y_{0}^{*}\right)-\frac{(\lambda-\alpha)^{2}}{2 \kappa \lambda}\left(y_{2}-y_{0}^{*}\right)^{2} .
$$

Maximizing (8) with respect to $y_{2}$ yields the equilibrium value

$$
y_{2}^{*}=y_{0}^{*}\left(1+\frac{\omega \kappa}{(1-\omega)^{2}}\right) \text {. }
$$

As in the base case, adoption by the complete market takes place when $\omega \rightarrow 1 \Leftrightarrow \alpha \rightarrow \lambda$. This is in contrast to $y_{1}^{*}$ derived in the preceding section, which diverges already for smaller values $\left(\right.$ at $\left.\omega \rightarrow(\sqrt{\kappa}+1)^{-1}\right)$. The reason for this difference is that, in the present model, consumers in $\left[y_{0}^{*} ; \hat{y}_{2}\right]$ do not act as sponsors, while in the earlier model those in $\left[y_{0}^{*} ; \hat{y}_{1}\right]$ do. Figure 3 illustrates equation (9). Also under the assumptions made here a considerable network growth can be attained. For example, with $\omega=0.45$ and $\kappa=2$, the network grows by a factor of 4 compared to the base case.

\subsection{Small Groups of Sponsors}

The assumption of coordination between all early adopters made above is problematic. In general, there will be an incentive to shirk. In this and the following section we show how the public good problem inherent in working to grow the network can be overcome.

In contrast to the preceding sections we do not consider the aggregate utility of certain consumer segments. Instead, we consider a single new adopter who receives sponsoring by a small group of sponsors. This group is assumed to be of size $n$, where the limiting case $n=1$ is particularly relevant. The logic behind the assumption of a small group is that its members can coordinate their joint sponsoring effort much more easily than the

\footnotetext{
${ }^{14}$ After they have gone through the adoption process they might, in a third stage, act as sponsors themselves. However, we restrict our analysis here to two stages. An extension to three or more stages would of course be feasible. However, if one aims at making the temporal structure more realistic, then a more suitable choice would be to introduce continuous time instead of three or more stages.
} 


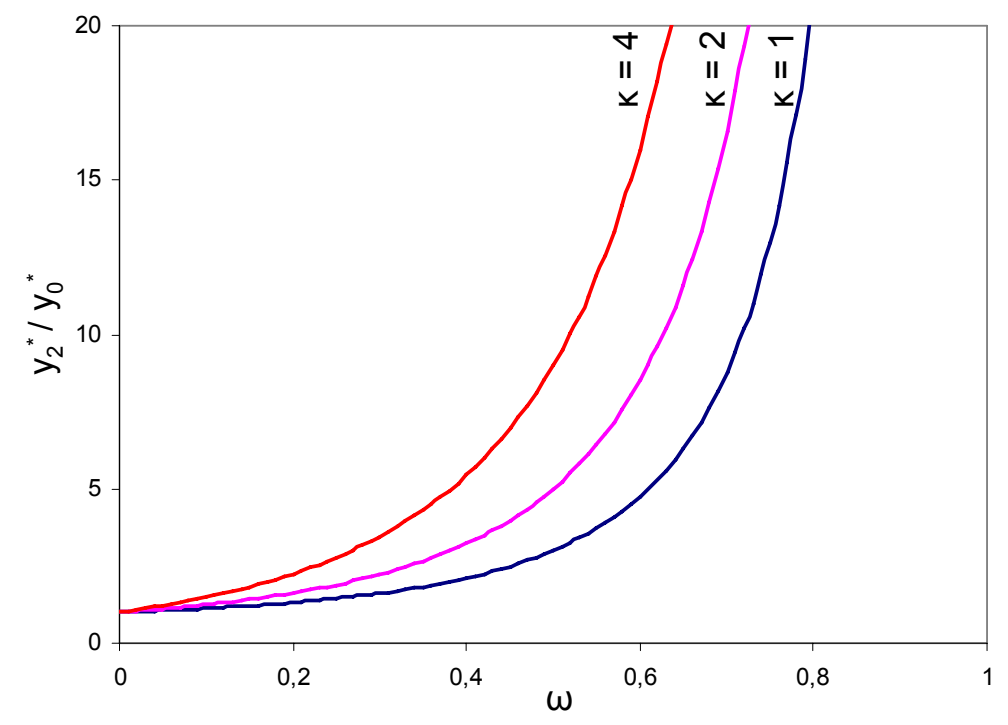

Figure 3: Relative network growth $y_{2}^{*} / y_{0}^{*}$ due to peer effect, see (9).

early adopter segment as a whole. In addition, monitoring to prevent shirking is easier in small groups. While free riding is still an issue (since all network members benefit equally when a certain group manages to recruit a new adopter), the group's action is rewarding for the group independent of what other adopters do.

Since we consider individual processes of user recruitment, we also model the temporal structure of network growth differently. We assume that, given the size $y$ of the network, sponsoring efforts are directed towards the marginal non-consumer since this person requires the lowest sponsoring effort. Recruiting of new network members then continues until, for the group of size $n$, cost and benefit of alluring one additional consumer offset each other.

The utility change $\Delta U(n)$ that a group of adopters of size $n$ attains when convincing one additional consumer to join the network (currently of size $y$ ) is given by

$$
\Delta U(n)=n(v(y+1)-v(y))+\frac{1}{\kappa}(u(y+1)+v(y+1)-p) .
$$

In the linear case, equation (10) becomes

$$
\Delta U(n)=n \alpha+\frac{1}{\kappa}\left(u_{0}-(\lambda-\alpha)(y+1)-p\right) .
$$

The group will keep sponsoring further adopters as long as $\Delta U(n)$ is positive. The marginal adopter, and thus the resulting network size, is then given by $\Delta U(n)=0$, which in the linear case yields

$$
y_{3}^{*}=\frac{u_{0}-p+n \alpha \kappa}{\lambda-\alpha}
$$




$$
=y_{0}^{*}+\frac{n \omega \kappa}{1-\omega}
$$

Equation (13) shows that, under the mechanism of peer influence assumed here, the network growth compared to the base case is not by a factor (as in the cases of $y_{1}^{*}$ and $y_{2}^{*}$ analyzed above), but by a fixed number of new participants. This fact has an important implication: A sizeable relative expansion of the network induced by the peer effect (as modeled here) is only possible for small networks. For example, with $\omega=0.5$ and $\kappa=3$, network participants banding together in groups of two can attain an overall growth of the network by six members. For a network with a base case equilibrium size of, e.g., tenthousand this increase is negligible. In contrast, for a network whose base case equilibrium size equals 10 an increase by six makes for a quite impressive growth of 60 percent. And even if coordination in small groups is not feasible (i.e., $n=1$ ), still a growth by 30 percent can be achieved.

Given the examples of ebay and Skype mentioned earlier, a base case network size of 10 may appear absurdly small. However, even though both goods - or rather, services - have been adopted by many millions of users, the effective network size in the case of Skype is much smaller. While we are lacking precise data, it is safe to assume that Skype's user base consists of a large number of small (sub-)networks defined by a high communication intensity among its members, and a low communication intensity between its members and non-members. ${ }^{15}$ While it is true that also ebay's user base will be divided into sub-networks, e.g., of individuals trading diving equipment, these sub-networks will in general be much larger than in the case of Skype.

\subsection{Comparison of Models}

From the first to the third of the models presented in this section, our assumptions have become more realistic. The model presented in 3.2 led, in the linear case, to a network size of $y_{1}^{*}(5)$. It serves as a benchmark case, illustrating the implications of peer influence. However, this model made the unrealistic assumption of an adoption-maximizing re-distribution of the surplus generated by the network growth from the base case size $y_{0}^{*}(3)$ to $y_{1}^{*}$. The second model introduced a more realistic, two-stage timing structure. It assumed that only early adopters act as sponsors to later adopters, and that sponsors maximize their utility (as opposed to network size). The final model then addressed the public good problem inherent in coordination between sponsors, by assuming that small groups of sponsors coordinate to allure the respective marginal non-consumer. In addition, it considers all acts of sponsoring as consecutive. Hence, it makes both the sponsoring mechanism and its timing structure even more realistic. Due to the fixed size

\footnotetext{
${ }^{15}$ Of course, our model is a simplification of this scenario by allowing only two levels of communication intensity: either two individuals belong to the same network or they do not. We also abstract from the fact that some individuals will belong to more than one sub-network.
} 
of the sponsoring group also the network growth achieved, from $y_{0}^{*}$ in the base case to $y_{3}^{*}$, is by a fixed number of adopters, not (as for $y_{1}^{*}$ and $y_{2}^{*}$ ) by a factor.

Comparing the equilibrium network size between the three models we find $y_{1}^{*}$ larger than $y_{2}^{*}$ and, unless the base case network size $y_{0}^{*}$ is rather small, $y_{2}^{*}$ larger than $y_{3}^{*}$. Given that the additional assumptions introduced in the second and the third model restrict the peer effect mechanism, this finding comes as no surprise. More interesting is the qualitative difference that the first and the second model lead to an increase in network size by a multiplicative factor, while the third model leads to an increase by an additive constant. Since the third model is both the most conservative and the most realistic one, we use it for all further analysis.

\section{Monopoly: The Seller's Perspective}

From a monopolistic seller's perspective, the peer effect is clearly advantageous. The question arises if and how the seller of the network good should take the peer effect into account when devising its strategy. In this section, we address the aspects of price setting and subsidies.

\subsection{Price Setting}

Consider, as above, a monopolistic seller of a network good facing a peer effect as modelled in Section 3.4. The seller has to bear a fixed $\operatorname{cost} F$ of entering the market, and a variable cost $c$ per unit. In the base case, the seller's profit $\Pi_{0}(p)$ obtains as $\Pi_{0}(p)=(p-c) y_{0}^{*}-F$, which in the linear model becomes

$$
\Pi_{0}(p)=(p-c) \frac{u_{0}-p}{\lambda-\alpha}-F
$$

The profit-maximizing price is

$$
p_{0}^{*}=\frac{1}{2}\left(u_{0}+c\right)
$$

leading to profits of

$$
\Pi_{0}^{*}=\frac{\left(u_{0}-c\right)^{2}}{4(\lambda-\alpha)}-F
$$

We now take the peer effect into account. When the peer effect is exerted by sponsor groups of size $n$, an equilibrium network size of $y_{3}^{*}>y_{0}^{*}$ obtains (13). In the linear case, profit function, equilibrium price, and equilibrium profit are

$$
\begin{aligned}
\Pi_{3}(p) & =(p-c) \frac{u_{0}-p+n \alpha \kappa}{\lambda-\alpha}-F, \\
p_{3}^{*} & =\frac{1}{2}\left(u_{0}+n \alpha \kappa+c\right),
\end{aligned}
$$




$$
\Pi_{3}^{*}=\frac{\left(u_{0}+n \alpha \kappa-c\right)^{2}}{4(\lambda-\alpha)}-F
$$

That is, compared to the base case the presence of the peer effect leads to higher prices and, less surprising, higher profits.

The equations derived here allow comparing the equilibrium network sizes under endogenous pricing (while the comparison of $y_{0}^{*}$ and $y_{3}^{*}$ so far was made at given prices $p$ ). We denote equilibrium network size in model $i$ under endogenous pricing by $y_{i}^{* e}$.

$$
\begin{aligned}
y_{0}^{* e} & =\frac{u_{0}-c}{2(\lambda-\alpha)} \\
y_{3}^{* e} & =\frac{u_{0}+n \alpha \kappa-c}{2(\lambda-\alpha)} \\
& =y_{0}^{* e}+\frac{n \omega \kappa}{2(1-\omega)} .
\end{aligned}
$$

Equation (22) shows that, although the price increase by $p_{3}^{*}-p_{0}^{*}=n \alpha \kappa / 2$ mitigates the network expansion due to the peer effect, the equilibrium network size under endogenous pricing, $y_{3}^{* e}$, is still larger than that in the absence of a peer effect, $y_{0}^{* e}$. More precisely, the relative increase from $y_{0}^{* e}$ to $y_{3}^{* e}$ equals $n \omega \kappa /(2(1-\omega))$, i.e., it is half the size that under given price $p$ from $y_{0}^{*}$ to $y_{3}^{*}$ (cf. equation $(13)$ ).

\subsection{Subsidies by the Network Provider}

When all early adopters coordinate to exert peer influence, a large network growth compared to the base case can be realized (see Sections 3.2 and 3.3). However, the public good problem described earlier obviates this outcome. A potential solution, apart from coordination within small groups, is that the network provider offers incentives for exerting peer influence. We analyze this solution in the following.

We assume that, after adoption as in the base case has taken place, the seller of the network good offers a subsidy of $s$ to each user who recruits a new adopter. In analogy to equation (10) for $n=1$, the utility change for a sponsor receiving the subsidy for growing 
the network from $y$ to $y+1$ equals $^{16}$

$$
\Delta U_{s}=v(y+1)-v(y)+s+\frac{1}{\kappa}(u(y+1)+v(y+1)-p) .
$$

In the linear case, equation (23) becomes

$$
\Delta U_{s}=\alpha+s+\frac{1}{\kappa}\left(u_{0}-(\lambda-\alpha)(y+1)-p\right)
$$

Network members will keep recruiting new adopters as long as their gain $\Delta U_{s}$ from doing so is positive. The resulting network size is then given by $\Delta U_{s}=0$, which in the linear case yields

$$
\begin{aligned}
y_{4}^{*} & =\frac{u_{0}-p+(\alpha+s) \kappa}{\lambda-\alpha} \\
& =y_{0}^{*}+\frac{(\omega+s / \lambda) \kappa}{1-\omega} .
\end{aligned}
$$

Given the price $p$, what subsidy $s^{*}(p)$ would maximize the seller's profit? For the linear case, we find

$$
s^{*}(p)=\frac{1}{2}(p-c-\alpha) .
$$

Endogenizing $p$ and using (27), we find for the profit-maximizing price and the corresponding subsidy:

$$
\begin{aligned}
p_{4}^{*} & =\frac{2\left(u_{0}+c\right)+\kappa(\alpha-c)}{4-\kappa} \\
s^{*}\left(p_{4}^{*}\right) & =\frac{u_{0}-c-(2-\kappa) \alpha}{4-\kappa}
\end{aligned}
$$

Thus, for not too large values of $\alpha$ and $\kappa$ a positive and finite subsidy is offered by the seller, which increases in $\left(u_{0}-c\right)$ and $\kappa$. The dependence on $\alpha$ is U-shaped, decreasing for $\kappa<2$ and increasing for $\kappa>2$.

\footnotetext{
${ }^{16}$ We conservatively restrict our analysis here to the case of sponsoring by single individuals, since coordination within a group of size $n>1$ plus receiving and distributing the subsidy seems a rather complex procedure. We also abstract from the problem facing the seller to distinguish between early adopters who adopt without subsidies, and later adopters requiring a (possibly subsidy-driven) peer influence. Next, we assume that the seller can not discriminate between network members who would exert peer influence also without receiving a subsidy (who exist most likely while the network size is still close to $y_{0}^{*}$ ) and those who would not. Finally, we assume that the seller can not discriminate between network members requiring a large subsidy and those requiring only a small one.
} 


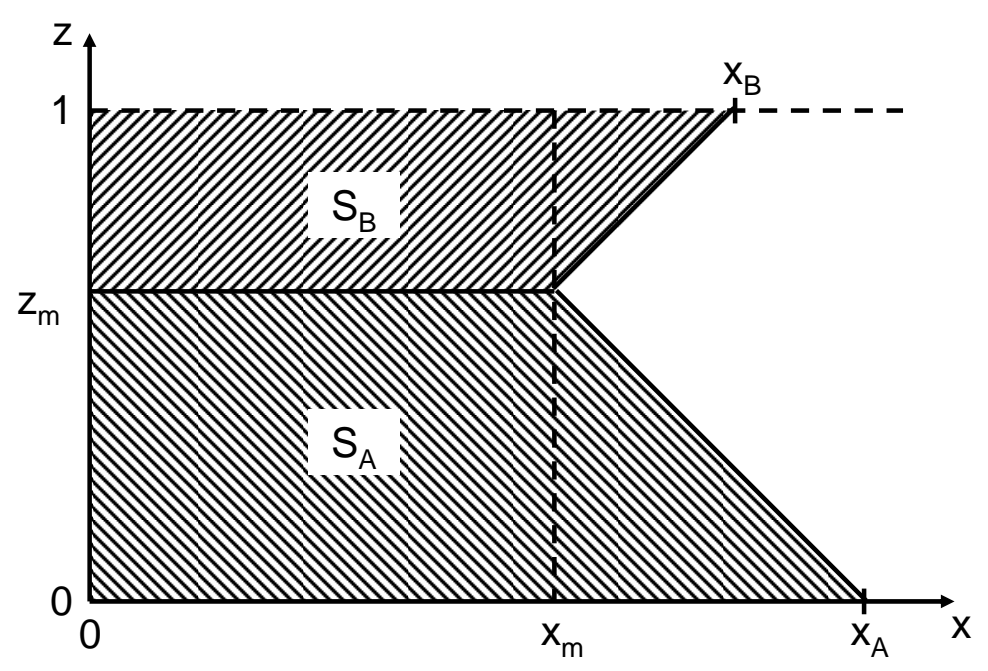

Figure 4: Market geometry in 2-dimensional model.

\section{Duopoly}

\subsection{Adoption Without Peer Influence}

We now turn to the case of duopolistic competition. We model duopoly in the following way: Consumers are uniformly distributed, with density one, on the strip $x \geq 0,0 \leq z \leq 1$ in the $(x, z)$ plane. The shops, or network goods, are located at $(0,0)$ (network A) and $(0,1)$ (network B), respectively. See Figure 4 for an illustration. We first analyze the base case, excluding the peer effect. The net utility for the consumer located at $(x, z)$ from purchasing good A, given an expected network size (i.e., adopter number) of $S_{A}^{e}$, is given by

$$
U_{A}(x, z)=u_{0}-\lambda(x+z)-p_{A}+\alpha S_{A}^{e} .
$$

An analogous equation holds for $U_{B}(x, z)$. We thus model "transportation cost" in the $z$ dimension, or the intensity of competition between $\mathrm{A}$ and $\mathrm{B}$, in the standard linear fashion introduced by Hotelling (1929). Generalizing to two dimensions, we measure the distance between the consumer at $(x, z)$ and each "shop" using a block metric. In order to keep the analysis tractable we restrict ourselves to the linear case from the outset. We will provide a qualitative discussion of the general case toward the end of this section. ${ }^{17}$

The position of the marginal consumers in $z$ direction is denoted by $z_{m}$. We assume that $u_{0}$ is large enough such that all consumers on the line from $(0,0)$ to $(0,1)$ are always served at equilibrium prices. When $z_{m}$ lies in the interior of the interval $[0 ; 1]$, then the

\footnotetext{
${ }^{17}$ The most obvious approach to integrating peer influence and competition might seem to be the standard, one-dimensional Hotelling model in which consumers are evenly distributed on the interval $[0,1]$ and shops are located at 0 and at 1 . However, this model fails to capture the most interesting implications that peer influence has in the context of competition, as will become clear below. Also extending the market to the full real line, while capturing the market-growing effect of peer influence, still does not yield a sufficiently realistic model.
} 
positions $x_{A}, x_{B}$ of the marginal consumers in $x$ direction (at $z=0$ and $z=1$, respectively, see Figure 4) relate to $z_{m}$ in the following way:

$$
z_{m}=\left(x_{A}-x_{B}+1\right) / 2
$$

Using (31) and $x_{m}$ as the position of the marginal consumer in $x$ direction at $z=z_{m}$, the market size $S_{A}$ for shop $A$ can be calculated from $x_{A}, x_{B}$ as

$$
S_{A}=z_{m}\left(x_{A}+x_{m}\right) / 2 \equiv\left(x_{A}-x_{B}+1\right)\left(3 x_{A}-x_{B}+1\right) / 8 .
$$

Necessary conditions for an FEE described by $\left(x_{A}^{*}, x_{B}^{*}\right)$ are that $U_{A}\left(x_{A}^{*}, 0\right)=0$ and $U_{B}\left(x_{B}^{*}, 1\right)=0$. Introducing $x_{S} \equiv x_{A}+x_{B}$ and $x_{D} \equiv x_{A}-x_{B}$ for the sum and difference of $x_{A}$ and $x_{B}$, the necessary conditions for an FEE can be expressed as

$$
\begin{aligned}
-\lambda x_{D}^{*}-p_{A}+p_{B}+\alpha x_{D}^{*} x_{S}^{*} / 2 & =0 \\
2 u_{0}+(\alpha / 2-\lambda) x_{S}^{*}-p_{A}-p_{B}+\alpha\left(x_{D}^{*}\right)^{2} / 4-\alpha / 4 & =0
\end{aligned}
$$

Solving this equation system explicitly leads to rather awkward terms which contain the roots of a third-order polynomial. However, for analyzing existence and stability of symmetric equilibria only small deviations from the symmetric case $x_{A}=x_{B}$ need to be analyzed. In this case, the quadratic term in (34) can be neglected as small in second order of $x_{D}^{*}$, and the equation system can be solved. The general expression for $x_{A}^{*}$ is given in the Appendix (44). For $p_{A}=p_{B}=p$, the solution is

$$
x_{A}^{*}=\frac{8 u_{0}-8 p-\alpha}{4(2 \lambda-\alpha)} .
$$

We now check if the above equation describes a stable equilibrium. ${ }^{18}$ Consider, at given and identical prices $p$, a small shift of the marginal consumer $z_{m}$ from the hypothesized equilibrium value of 0.5 to $0.5+\epsilon{ }^{19}$ This shift increases the market area $S_{A}$, which makes

\footnotetext{
${ }^{18}$ The derivation of equation (35) as well as the following stability analysis would not have required the general approach followed above; in particular, we could have set $p_{A}=p_{B}$ from the outset. The general formulation is needed for endogenizing the sellers' pricing decision (which is currently work in progress).

${ }^{19}$ In contrast to the classical one-dimensional Hotelling model, the marginal consumer indifferent between networks $A$ and $B$ is not unique. Since the market in our model is two-dimensional, we have a continuum of marginal consumers along the border between the market areas $S_{A}$ and $S_{B}$. Hence, when we consider defection of a marginal consumer, out of a hypothesized equilibrium state, from shop $B$ to shop $A$, it is not obvious "which" marginal consumer to choose. Our approach - to consider defection by a "strip of consumers" spanning the complete border between $S_{A}$ and $S_{B}$ - is appealing for reasons of symmetry. In addition, it correctly captures the intuition for the (realistic) case of discrete, "point-like" consumers: the larger the markets $S_{A}$ and $S_{B}$ (in our model: the longer the border between them), the smaller the expected minimum utility difference between $A$ and $B$ among the adopters of $B$, and the more likely defection of one adopter from one good to the other. This intuition would not be correctly captured if one modeled a defecting marginal consumer as a market segment of quadratic shape.
} 
adoption of the good more attractive and thus also leads to growth of $S_{A}$ in $x$ direction. This growth, from $x_{A}^{*}$ to $x_{A}^{*}+\delta$, can be calculated from the condition that the utility of the "new" marginal consumer at $\left(x_{A}^{*}+\delta, 0\right)$ equals zero:

$$
\begin{aligned}
0 & =u_{0}-\lambda\left(x_{A}^{*}+\delta\right)-p+\alpha(1 / 2+\epsilon)\left(x_{A}^{*}+\delta-1 / 4\right) \\
& \approx\left[u_{0}-\lambda x_{A}^{*}-p+\alpha\left(x_{A}^{*}-1 / 4\right) / 2\right]+\left[-\lambda \delta+\alpha \epsilon\left(x_{A}^{*}-1 / 4\right)+\alpha \delta / 2\right]
\end{aligned}
$$

From (36) to (37) the term proportional to $\epsilon \delta$ has been omitted since it is small in second order. The first bracket in (37) vanishes due to the definition of $x_{A}^{*}$. The second thus also vanishes, which allows to express $\delta$ as

$$
\delta=\epsilon \frac{\alpha\left(4 x_{A}^{*}-1\right)}{2(2 \lambda-\alpha)}
$$

Now the change in utility that the "new" marginal consumer at $(0,0.5+\epsilon)$ experiences can be calculated. Only if it is negative, the symmetric equilibrium candidate truly represents a (stable) equilibrium.

$$
U_{A}(0,1 / 2+\epsilon)=u_{0}-\lambda(1 / 2+\epsilon)-p+\alpha(1 / 2+\epsilon)\left(x_{A}^{*}-1 / 4+\epsilon \frac{\alpha\left(4 x_{A}^{*}-1\right)}{2(2 \lambda-\alpha)}\right.
$$

Collecting terms linear in $\epsilon$, and ignoring those quadratic in $\epsilon$, allows to deduce the condition for stability of the symmetric solution described by (35):

$$
4\left(u_{0}-p\right)<\lambda+\frac{(2 \lambda-\alpha)^{2}}{\alpha} \equiv \lambda\left(1+\frac{(2-\omega)^{2}}{\omega}\right)
$$

This result serves as the base case for our competition model.

\subsection{Adoption Under Peer Influence}

In contrast to the monopoly case, there are two types of marginal consumers in our duopoly model - those indifferent between goods $A$ and $B$, and those indifferent between one of the goods and the outside option. Peer influence is exerted on both of them. However, for those consumers along the border between the markets of $A$ and $B$ peer influence from both sides will cancel each other out. We thus only consider the marginal consumer in $x$ direction.

Restricting ourselves, for the time being, to exogenous and equal prices $p_{A}=p_{B}=p$, the symmetric equilibrium candidate $\tilde{x}_{A}^{*}$ is characterized by

$$
0=U_{A}\left(\tilde{x}_{A}^{*}, 0\right)+n \alpha \kappa
$$




$$
\equiv u_{0}-\lambda \tilde{x}_{A}^{*}-p+\alpha\left(\tilde{x}_{A}^{*}-1 / 4\right) / 2+n \alpha \kappa
$$

which leads to

$$
\tilde{x}_{A}^{*}=\frac{8 u_{0}-8 p-\alpha+8 n \alpha \kappa}{4(2 \lambda-\alpha)} .
$$

This equation is largely identical to (35), with $\left(u_{0}-p+n \alpha \kappa\right)$ instead of $\left(u_{0}-p\right)$.

Since the analysis follows the same steps as in the base case, we arrive at the stability condition in the case of peer influence by replacing $\left(u_{0}-p\right)$ in equation $(40)$ with $\left(u_{0}-\right.$ $p+n \alpha \kappa):$

$$
4\left(u_{0}-p\right)<\lambda+\frac{(2 \lambda-\alpha)^{2}}{\alpha}-4 n \alpha \kappa \equiv \lambda\left(1+\frac{(2-\omega)^{2}}{\omega}-4 n \kappa \omega\right)
$$

Condition (43) shows that the peer effect makes it more difficult to fulfill the stability condition. More precisely, there are parameter values at which a symmetric equilibrium exists absent the peer effect, but does not exist if the peer effect is present. The peer effect thus favors winner-take-all outcomes - the more, the higher the cost-leveraging factor $\kappa$, the size $n$ of sponsor groups, and the relative strength $\omega$ of the network externality.

Endogenizing the sellers' pricing decisions is currently work in progress. The basic trade-offs, however, can also be discussed verbally. In the monopoly case, the peer effect leads to higher prices. This is due to the fact that the seller partly skims off the increase in attractiveness (due to the peer effect) of her good. Also in duopoly, this effect should come to bear. On this other hand, this same increased attractiveness leads to more adoption and hence more marginal or close-to-marginal consumers, which should make competition tougher in duopoly. It depends on the parameter values which effect prevails.

\section{Empirical Study}

To confirm the main results of our model, we conducted an online survey with 373 participants. We asked the participants for the relative importance of the local installed base, the global installed base, and peer influence for their decision to adopt eBay and Skype, respectively. The survey took place between August 2nd and September 24th 2007. Most participants were students, with an average age of 26.6 years (median 25 years). 241 (65\%) were male. 277 (74\%) had adopted Skype, 291 (78\%) had used eBay (as a buyer, a seller, or both).

To find out about the relative importance of the three constructs, we compared each construct with the other two constructs by means of a semantic differential. Figure 6 in the Appendix shows the exact statements and the corresponding scales that we used. The results are clear-cut (see Figure 7). For the adoption of Skype, peer influence is considered the most important trigger, closely followed by local network effects. The 


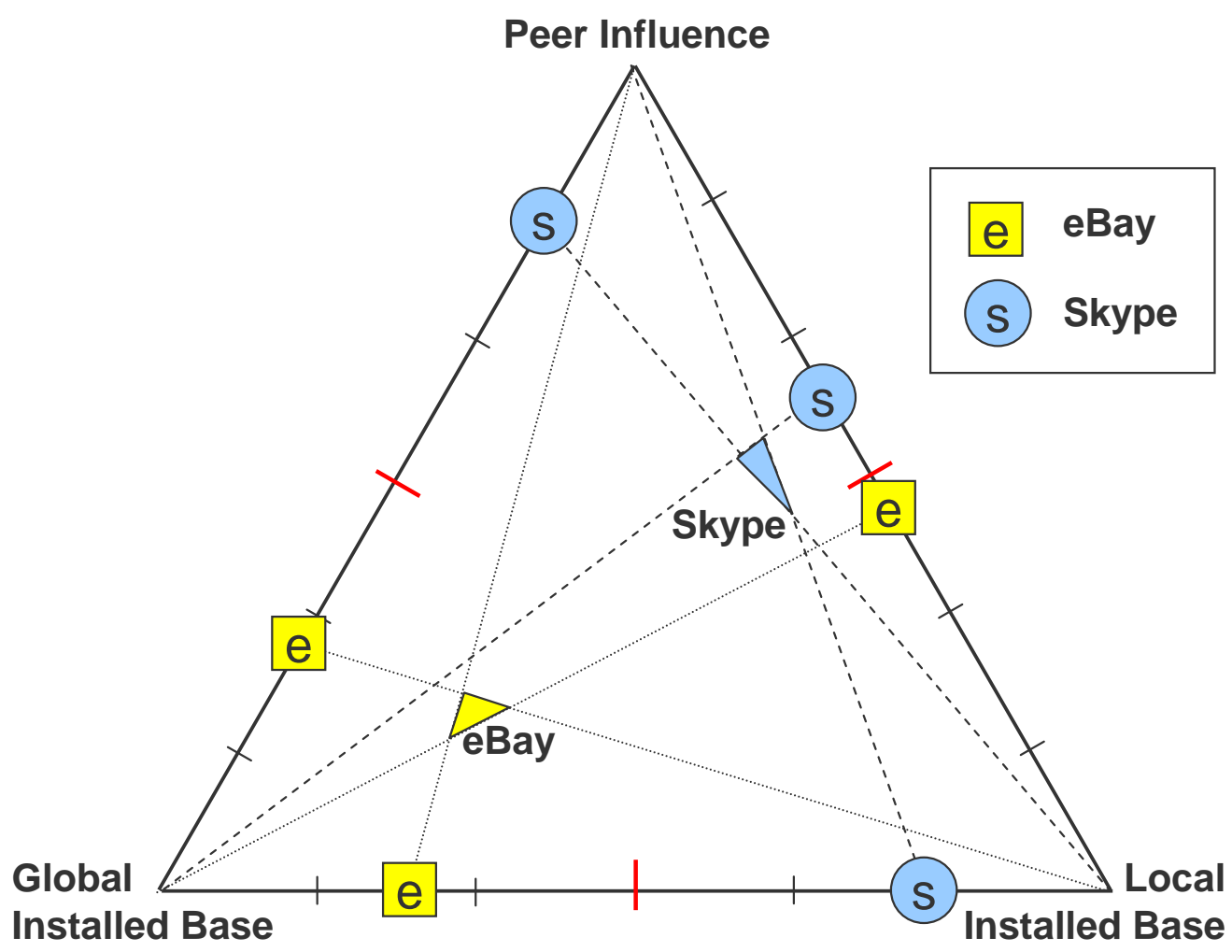

Figure 5: Relative importance of peer effect, local installed base, and global installed base for the adoption of Skype and eBay, respectively.

relevance of global network effects is nearly negligible. For the adoption of eBay the picture is completely reversed: now, global network effects are the most important trigger, with local network effects and peer influence both far behind and roughly equally unimportant. Figure 5 below illustrates and summarizes our results graphically. The high level of consistency of the ratings given by the survey participants is reflected in the three lines, for each of the two services, intersecting to a good approximation in a single point.

To further check the validity of the peer influence argument, we asked the participants about the number of friends which they persuaded to use Skype or eBay. Again, the answer is very clear and consistent with our model results. The Skype adopters persuaded on average 2.90 friends (median: 2) to also register with Skype, whereas the eBay adopters persuaded on average only 2.06 friends (median: 1 ) to also use eBay.

\section{Discussion}

Network effects have two implications. First, the larger a network, the more attractive it becomes for outsiders to join it. This installed base effect has been treated extensively in the literature. Second, growing the network by exerting peer influence on not-yetadopters is beneficial for network members, who can thus be expected to engage in it. On this peer effect, the extant literature is largely silent (Domingos and Richardson (2001) 
and Subramani and Rajagopalan (2003) being exceptions). While it will often be effected by word of mouth, the peer effect clearly stands out from common instances of word of mouth marketing by the type of motivation that drives the acting person - namely, continuous benefits due to internalization of a network externality.

Our game-theoretical model yields a number of findings. Most importantly, the distinction between two types of networks arises endogenously: local, personal, and small networks on the one hand, and global, anonymous, and large networks on the other hand. In the latter type of networks, the peer effect is impeded by a public good problem. This public good problem is overcome in small networks, and the peer effect can lead to a considerable relative growth compared to the base case absent peer influence.

The empirical study provided clear support for our model findings. For Skype - the adoption of which largely replicates existing social ties - peer influence and the local installed base effect trigger adoption. Local, small, and personal networks result (which are, of course, linked to some extent by weak ties). For eBay, which users adopt with the purpose of creating new ties, the global installed base effect dominates, leading to a global, large, and anonymous network.

Local network externalities are modeled by heterogeneity between dyads of users of the network good (e.g., Cowan and Miller 1998). This heterogeneity leads to clusters of individuals who value their common membership in the local network highly, but put a lower value on being linked to others. This local network corresponds to our (total) network when its size is small. A global network of loosely connected local networks then corresponds, in our model, to a patchwork of many independent small networks. This interpretation explains the seeming paradox that, as we have shown, the peer effect favors winner-take-all outcomes, while local network externalities tend to produce nonstandardized results (Cowan and Miller 1998; Jonard and Yildizoglu 1998): the peer effect favors winner-take-all outcomes within each local network, but conflicts in no way with different outcomes in different local networks. It seems an interesting question, though, in how far the peer effect in the presence of weak ties between local networks can indeed also favor winner-take-all outcomes spanning more than one local network. 


\section{Appendix}

\section{Symbols}

$c$ Variable cost of providing the network good

$F$ Fixed cost of providing the network good

$n$ Size of small sub-network which coordinates to recruit a new adopter

$s$ Subsidy paid by seller to a network member recruiting a new adopter

$u_{0}$ Constant in the linear model of $u(x): u(x)=u_{0}-\lambda x$

$u(x)$ Stand-alone utility (i.e., excluding network externalities) which consumer $x$ derives from the good

$v(y)$ Utility that each adopter derives due to the network effect when the network size equals $y$

$y_{i}^{*}$ Marginal adopter in equilibrium, in model $i$

$\alpha$ Slope parameter in the linear model of $v(y): v(y)=\alpha y$

$\kappa$ Cost leveraging factor: When exerting peer influence on some not-yet-adopter creates a cost, the resulting benefit for the wooed individual equals $\kappa$ times the cost.

$\lambda$ Slope parameter in the linear model of $u(x): u(x)=u_{0}-\lambda x$

$\omega \omega \equiv \alpha / \lambda$ measures the relative strength of the network externality

\section{Calculation of FFE in Duopoly}

The marginal consumer $x_{A}^{*}$ in an FEE with exogenous prices, close to a symmetric situation (i.e., $p_{A} \approx p_{B}$ ), is given by:

$$
\begin{aligned}
x_{A}^{*}= & \frac{1}{4}\left(-4 \alpha \lambda+8 \lambda^{2}-8 \alpha u_{0}+4 \alpha p_{A}+4 \alpha p_{B}+\alpha^{2}\right)^{-1}(2 \lambda-\alpha)^{-1} \\
& \cdot\left(16 \alpha^{2} p_{A}-48 \alpha \lambda p_{A}+64 p_{A} \lambda^{2}+16 \alpha \lambda p_{B}+32 \alpha \lambda u_{0}-64 u_{0} \lambda^{2}+64 \alpha u_{0}^{2}-64 \alpha u_{0} p_{A}\right. \\
& \left.-64 \alpha u_{0} p_{B}-16 \alpha^{2} u_{0}+16 \alpha p_{A}^{2}+32 \alpha p_{A} p_{B}+16 \alpha p_{B}^{2}-4 \alpha^{2} \lambda+8 \alpha \lambda^{2}+\alpha^{3}\right)
\end{aligned}
$$




\section{Peer Influence vs. Local Network Effects}

My final decision to start using Skype (Ebay) was triggered by

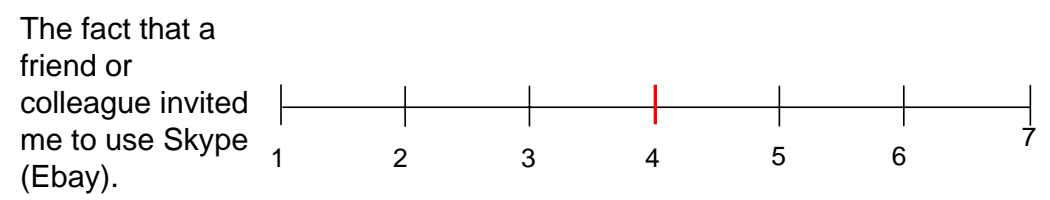

The number of users of Skype (Ebay) among my friends or colleagues.

\section{Global Network effects vs. Local Network Effects}

My final decision to start using Skype (Ebay) was triggered by

The total number of Skype (Ebay) users.

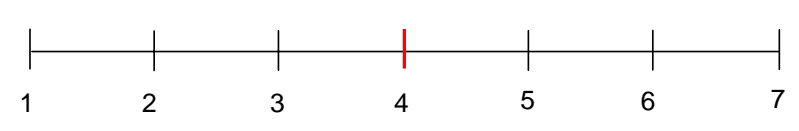

The number of

Skype (Ebay) users among my friends or colleagues.

\section{Peer Influence vs. Global Network Effects}

My final decision to start using Skype (Ebay) was triggered by

The fact that a friend or

colleague invited me to use Skype (Ebay).

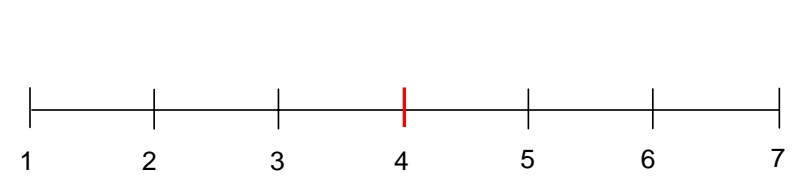

The total number of users of Skype (Ebay).

Figure 6: Semantic differentials as presented to respondents. 
Peer Influence vs. Local Network Effects

\begin{tabular}{|l|c|c|c|c|c|c|}
\hline & $\mathbf{N}$ & Min. & Max. & Mean & Std. dev. & $\begin{array}{c}\text { t-test on equality } \\
\text { of means }\end{array}$ \\
\hline Skype & 277 & 1 & 7 & 3.41 & 1.89 & \multirow{2}{*}{$\mathrm{p}<0.001$} \\
\hline Ebay & 291 & 1 & 7 & 4.26 & 1.54 & \multirow{2}{*}{ Eb } \\
\hline
\end{tabular}

Global vs. Local Network Effects

\begin{tabular}{|l|c|c|c|c|c|c|}
\hline & N & Min. & Max. & Mean & Std. dev. & $\begin{array}{c}\text { t-test on equality } \\
\text { of means }\end{array}$ \\
\hline Skype & 277 & 1 & 7 & 5.81 & 1.56 & \multirow{2}{*}{$\mathrm{p}<0.001$} \\
\hline Ebay & 291 & 1 & 7 & 2.67 & 1.74 & \\
\hline
\end{tabular}

Peer Influence vs. Global Network Effects

\begin{tabular}{|l|c|c|c|c|c|c|}
\hline & N & Min. & Max. & Mean & Std. dev. & $\begin{array}{c}\text { t-test on equality } \\
\text { of means }\end{array}$ \\
\hline Skype & 277 & 1 & 7 & 2.14 & 1.45 & $p<0.001$ \\
\cline { 1 - 6 } Ebay & 291 & 1 & 7 & 5.17 & 1.79 & \\
\hline
\end{tabular}

Figure 7: Responses to semantic differential questions. 


\section{References}

Abrahamson, Eric \& Lori Rosenkopf. 1997. Social Network Effects on the Extent of Innovation Diffusion: A Computer Simulation. Organization Science 8(3): 289-309.

Arndt, Johan. 1967. Role of Product-Related Conversations in the Diffusion of a New Product.Journal of Marketing Research IV(August): 291-95.

Arthur, Brian W. 1989. Competing Technologies, Increasing Returns, and Lock-In by Historical Events. The Economic Journal 99(March): 116-31.

Block, Joern H. \& Philipp Köllinger. 2006. Peer Influence in Network Markets: An Empirical Investigation. http://ssrn.com/abstract=886719.

Brynjolfsson, Erik \& Chris F. Kemerer. 1996. Network Externalities in Microcomputer Software: An Econometric Analysis of the Spreadsheet Market. Management Science 42(12): 1627-47.

Cowan, Robin and John H. Miller. 1998. Technological Standards with Local Externalities and Decentralized Behaviour. Journal of Evolutionary Economics 8(3): 285-96.

Church, Jeffrey \& Ian King. 1993. Bilingualism and Network Externalities. Canadian Journal of Economics 26(2): 337-45.

David, Paul A. 1985. Clio and the Economics of QWERTY. American Economic Review 75(2): 332-7.

Deroïan, Frédéric. 2002. Formation of social networks and diffusion of innovations. Research Policy 31: 835846.

Domingos, Pedro \& Matt Richardson. 2001. Mining the Network Value of Customers. Proceedings of the seventh ACM SIGKDD international conference on knowledge discovery and data mining, San Francisco, California: 57 - 66.

Economides, Nicholas \& Charles Himmelberg. 1995. Critical Mass and Network Size with Application to the US FAX Market. Stern School of Business Working Paper 95-11.

Farrell, Joseph \& Garth Saloner. 1985. Standardization, Compatibility, and Innovation. Rand Journal of Economics 16(1): 70-83. 
Farrell, Joseph \& Garth Saloner. 1986. Installed Base and Compatibility: Innovation, Product Preannouncements, and Predation. American Economic Review 76(5): 940-55.

Jonard, Nicolas \& Murat Yildizoglu. 1998. Technological Diversity in an Evolutionary Model with Localized Learning and Network Externalities. Structural Change and Economic Dynamics 9(1): 35-51.

Gandal, Neil. 1994. Hedonic Price Indexes for Spreadsheats and an Empirical Test for Network Externalities. Rand Journal of Economics 25(1): 160-70.

Huang, Ching-I. 2004. Peer Effects and Consumption Behavior in Interconnected Networks. Working Paper, Northwestern University.

Katz, Michael L. \& Carl Shapiro. 1985. Network Externalities, Competition, and Compatibility. American Economic Review 75(3): 424-40.

Katz, Michael L. \& Carl Shapiro. 1986. Technology Adoption in the Presence of Network Externalities. Journal of Political Economy 94(4): 822-41.

Katz, Michael L. \& Carl Shapiro. 1992. Product Introduction with Network Externalities. The Journal of Industrial Economics 40(1): 55-83.

Koski, Heli. 1999. The Installed Base Effect: Some Empirical Evidence from the Microcomputer Market. Economics of Innovation and New Technology 8: 273-310.

Koski, Heli \& Tobias Kretschmer. 2004. Survey on Competing in Network Industries: Firm Strategies, Market Outcomes, and Policy Implications. Journal of Industry, Competition and Trade 4(1): 5-31.

Lazarsfeld, Paul F., Bernard Berelson \& Hazel Gaudet. 1944. The People's Choice: How the Voter Makes up His Mind in a Presidential Election. New York: Columbia University Press.

Liebowitz, Stan. 2002. Re-Thinking the Network Economy: the True Forces that Drive the Digital Marketplace. New York: American Management Association.

Liebowitz, Stan J. \& Stephen E. Margolis. 1990. The Fable of the Keys. Journal of Law \& Economics 33(April): 1-25. 
Majumdar, Sumit K. \& S. Venkataraman. 1998. Network Effects and the Adoption of new Technology: Evidence from the U.S. Telecommunications Industry. Strategic Management Journal 19(11): 1045-62.

Rysman, Marc. 2004. Competition between Networks: A Study of the Market for Yellow Pages. Review of Economic Studies 71(2): 483-512.

Saloner, Garth \& Andrea Shepard. 1995. Adoption of Technologies with Network Effects: An Empirical Examination of the Adoption of Automated Teller Machines. Rand Journal of Economics 26(3): 479-501.

Shankar, Venkatesh \& Barry L. Bayus. 2003. Network Effects and Competition: An Empirical Analysis of the Home Video Game Industry. Strategic Management Journal 24(4): 375-84.

Subramani, Mani R. \& Balaji Rajagopalan. 2003. Knowledge-sharing and influence in online social networks via viral marketing. Communications of the ACM 46(12): 300-307.

Sundararajan, Arun. 2005. Local Network Effects and Complex Network Structure. Working Paper, New York University. 